Journal of Computer Science 3 (2): 113-118, 2007

ISSN 1549-3636

(C) 2007 Science Publications

\title{
Gas Turbine Ontology for the Industrial Processes
}

\author{
${ }^{1}$ F.Z. Laallam and ${ }^{2}$ M. Sellami \\ ${ }^{1}$ Faculty of Sciences and Sciences of Engineering, Department of the Math and Data Processing \\ University of Ouargla, Road of Ghardaïa, 30000 Ouargla Algeria \\ ${ }^{2}$ Institute of Data Processing, LRI, Badji Mokhtar University, Annaba, Algeria
}

\begin{abstract}
The activity of supervision and control of the industrial processes is a very complex spot and require a great experiment because of the dynamic characteristics of the process. This experiment was acquired with the passing of years. What makes departure of an expert in retirement a great loss of the know-how. The problem thus consists to capture this know-how and allows experiment to be cumulate with an aim of construction of an enterprise memory. We propose an approach based on ontology to capture this know-how. In the dynamic situations are distinguished three classes from situations: situations of normal walk, situations of degraded walk and situations of incidental walk. The work presented in this article, was developed in the division of production at SONATRACH. It relates to the supervision and the control of the industrial process of a compressor station witch constitutes a typical case of dynamic situation. Among the three classes of quoted dynamic situations, we concentrate on the situations of degraded walk. These situations of nature different compared to the usual situations from normal walk, subject the operator to a workload at the same time complex and stressing. The work presented in this article enters within the framework of a doctoral project whose the principal objective was the development of an intelligent system of expertise and of decisionmaking aid in the domain of the industrial maintenance for the compression stations. It relates to ontological engineering and more particularly the use of ontologies in the knowledge-based systems. We try in this work to build an ontology concerning the domain of the industrial maintenance. This ontology was not operational yet because it does not included mechanisms of reasoning. It was independent of any context of use.
\end{abstract}

Key words: Ontology, industrial maintenance, knowledge management, protégé-2000

\section{INTRODUCTION}

A compression station is a unit or a series of compressors witch that aspires the fluid to a rather low pressure and rejects this fluid with a pressure definitely higher. Its role is thus to reduce volume and to raise the pressure of gas. This compression is operated in two times by the two groups which make the station. The first group consists of a gas compressor on two floors and the second comprises two distinct compressors with gases assembled on the same tree. These compressors are pulled by turbines. The activity of supervision and control of such industrial processes is a very complex spot and require a great experiment. This experiment risks to be lost with each departure of an employee on the one hand and to be dispersed between several distant experts on the other hand, what deprives the company to benefit from this experiment and to exploit it in an effective way.

Our work enters in the context of a shared system where experts of domain need to share and exchange knowledge, from a distance, in an aim of collaboration for the assistance in the diagnosis. What allows, on the one hand, to gather competences and on the other hand to have an experience feedback with an aim of capitalizing know-how.

To communicate, the agents must thus share an ontology. So in order to be a shared knowledge, an ontology must be explicit and be expressed in a language or a shared formalism.

In IA, one calls ontology the specification of the objects, concepts, classes, functions and relations of a domain independently of a particular application like the semantic networks and the conceptual graphs. They are used by people, data bases and applications needing to share information relating to a domain ${ }^{[1]}$. Ontology is thus the support of the acquisition of knowledge and it is also a useful tool to interface the software agents and the human agents. The work presented in this article consists of the conceptualization of ontology in the domain of the industrial maintenance. This ontology is developed for two reasons:

* To allow the agents of maintenance to share the common comprehension of the structure of information concerning gas turbines and their maintenance.

Corresponding Author: $\quad$ F.Z. Laallam, Faculty of Sciences and Sciences of Engineering, Department of the Math and Data Processing, University de Ouargla, Road de Ghardaïa, 30000 Ouargla Algeria, Tel: 078538715 
* To allow the re-use of the know-how on this domain.

Model based on ontologies: The models of representation of knowledge used in ontological engineering can be gathered according to conceptual paradigms' which they reify. They are thus distinguished:

* Models based on Frames ${ }^{[2]}$;

* Models based on Logics of Description;

* Models of the Conceptual Graphs.

The model of frames: The model of Frames was initially proposed, like language of representation of ontologies, by T. GRUBER. The principle of this model is to decompose knowledge into classes (or frames) which represent the concepts of the domain. To a frame, a certain number of attributes (slots) is attached, each attribute can take its values among a whole of facets (facets). Another way of presenting these attributes is to regard them as binary relations between classes whose first argument is called domain and the second range. Instances of the classes, corresponding to the extension of each concept, can be added, as well as functions which are particular types of relations binding a whole of classes to a computed value starting from the values of the attributes of the classes. The specification of conceptual properties of the attributes (or relations) resorts to formulas of first order logic. The semantics of subsumption is purely extensional: a frame F1 is more specific than a frame F2 if any instance of F1 is instance of F2. Several languages bases on frames exist. Let us quote as example: F-Logic which is the most known example of operational language containing frames.

Knowledge Interchange Format (KIF) is an example of language, nonoperational, implementing the model of the frames in first order logic for the representation of knowledge. Protégé2000 is another example of language, nonoperational, implementing the model of the frames. It uses the model of knowledge $\mathrm{OKBC}$ as bases for its proper model.

Logics of description: Logics of Description (DLL) combine intentional and extensional representations of knowledge:

* TBox ( $\mathbf{T}$ for terminological) contains the declarations of the organized conceptual primitives of concepts and roles (binary relations between concepts endowed with a domain and a range similar to the attributes of the frames). These declarations describe the properties of the concepts and the roles and thus constitute an intentional definition of knowledge;

* ABox (A for assertional) contains the declarations of individuals, instances of the concepts defined in TBox.
* The specification of properties of the concepts and roles uses ensemblist and logics constructors.

* Several models of DLL can be used according to the constructors necessary to modeling. The fact of using all the constructors or only one part of them operates on the decidability and the complexity of calculations of reasoning. Loom and CLASSIC are examples of operational models implementing this model. It is moreover used in the languages of representation of knowledge OIL and OWL developed for the Web.

The model of the conceptual graphs: Introduced by J. SOWA at the beginning of the Eighties, the model of the Conceptual Graphs (CG) belongs to the family of the semantic networks. The semantic networks model the knowledge in the form of graphs; nodes are associated to concepts and edges to relations. This model lends itself well to pictorial displays of knowledge but it presents certain particular problems of representation.

The model of CGs decomposes into two parts:

* A terminological part dedicated to the conceptual vocabulary of knowledge to represent, i.e. types of concepts, types of relations and instances of the types of concepts. This part corresponds to the representation of the conceptual model but also integrates knowledge on the hierarchic of the types of concepts and relations;

* An assertional part dedicated to the representation of the assertions of the domain knowledge studied ${ }^{[8]}$.

Construction of an ontology: Sharing common understanding of the structure of information among people or software agents is one of the more common goals in developing ontologies ${ }^{[6]}$ thus the role of an ontology is to consign a set of definitions of terms which corresponds to a conceptualization shared by the actors of a domain. The ontology contains the terminological primitives of the domain (the conceptual vocabulary structured in a set of concepts and a set of existing relations between these concepts) as well as the semantics of handling of these primitives expressed using axioms.

A concept is characterized by: a term, an extension (objects or instances of the concept handled through this concept) and an intension (the whole of the properties specifying the semantics of the concept);

A relation is characterized by: a term, an extension and an intension. It is used to describe a relationship among two or more terms. If a relation represents a relationship between only two terms, it is called a slot or a binary relation. If the relation describes a relationship among $\mathrm{n}$ terms such that there is a unique nth term corresponding to any set of the first n- 1 terms, then the relation is called a function ${ }^{[7]}$. 
An axiom is a sentence in first order logic that is assumed to be true without proof. In practice, we use axioms to refer to the sentences that cannot be represented using only slots and values on a frame. They are specific in ontologies and distinguish them from the thesauruses, who present only terminologies. The axioms can represent common properties related on the concepts and the relations. These properties are called "diagrams of axiom". Example: the property of subsumption (the relation "sort-of" between concepts or relations); generics of concept (concept generic or abstract which does not admit an instance).

Certain axioms appear in all the formalisms of representation of knowledge used to describe ontologies. Properties specific to the domain knowledge considered can also appear and included in ontology.

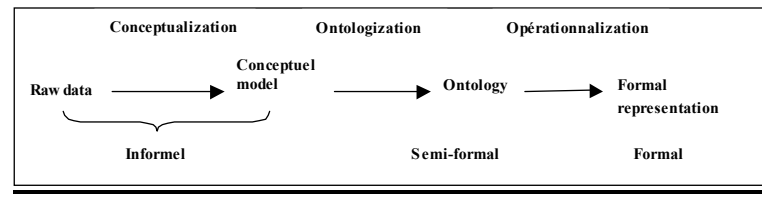

Fig. 1: Process of construction of an exploitable ontology within a knowledge-based system

Raw data, constituting a corpus (expressed a priori in natural language), integrates all knowledge of the domain which one wishes to formalize.

Step 1: Conceptualization: It consists in identifying precisely, starting from the corpus (a set of documents generally expressed in natural language which must cover the whole of the domain knowledge considered), conceptual objects specific to the considered domain (concepts, relations and axioms). An informal conceptual model then is obtained (because it is expressed in natural language) or an informal ontology. This model is used as support with the co-operative work of construction of ontology between the actors of maintenance. It constitutes moreover a privileged mean to diffuse ontology near people who would wish to reuse it for their own application or research tasks.

Step 2: Ontologization: It consists of a partial formalization of the conceptual model, without loss of information. It is a question of transcribing knowledge in a language or paradigm of representation of ontology (the Frame model, the entity-relation model, the model of conceptual graph or semantic network...).

Step 3: Operationnalization: It consists of the integration of knowledge in a knowledge-based system. This step consists in the complete formalization of the ontology obtained previously within the framework of a formal and operational language of representation of knowledge.
Whereas the construction of ontologies is the object of many researches, the operational use of ontologies was still few studied ${ }^{[3]}$.

\section{Description of our ontology: Study of case}

Presentation of the real system: Our process is a compressor station among other similar stations. Several components such as: turbines, compressor, balloons of torches... compose this station. The suspension of one of them following a breakdown can produce enormous damage for the company. The separation and treatment unit receives oil and crude gas on behalf of several areas (satellite) for their carrying out the operation of separation. This operation is based on the compression of the compressor which turns thanks to the turbine and the effect of heat. This implies that if the treatment unit breaks down, all the rough one coming from the satellite will be lost and directed towards the torches for a combustion in the case of a gas et in terrestrial surface in the case of oil, this to preserve the continuous operating condition for the satellites.

The breakdown of the turbine leads thus to crises on the level of the consumption of gas, oil and electricity generated by the turbine, thus the cost of the material is very high especially that most of repairs and the revisions are carried out by means of strange teams. All these consequences show the need for having a tool of assistance to accumulation of all the case of breakdowns to envisage them then to avoid them. It is about predictive maintenance.

We are interested in our work on the control of the gas turbine. These turbines have a cardinal importance in the production process and their costs of maintenance are very expensive.

\section{The gas turbine}

Description: The gas turbine is a motor machine with rotary movement and internal combustion, provided with a compressor with air and a combustion chamber able to produce a fluid under pressure and with very high temperature. This fluid, while slackening in the stages of the turbine, releases the mechanical energy to the external.

The turbine is made up of three principal parts:

1. An axial compressor: the principal function of the compressor is to compress the atmospheric air with a higher pressure.

2. Combustion chambers: the compressed air coming from the compressor is mixed with fuel and the mixture is lit. The product of this combustion is a hot gas vein with high pressure.

3. The wheel of the turbine: the hot gases with high pressure slacken by producing work to actuate the compressor of the turbine, on the one hand, and on the other hand to actuate the load. 
Functioning: The atmospheric air, aspired by the axial compressor, is compressed then driven back in the combustion chamber where the fuel is introduced; the desired mixture (compressed air and gas under pressure) is obtained.

A spark provided by candle causes combustion. The heat produced in the combustion chamber and the energy released by the combustion product are directed towards the first wheel of the turbine where this thermal energy is transformed into mechanical energy.

Part of the power developed by the turbine is used for the drive of the axial compressor (after its uncoupling of the engine or turbine of launching). The other part of the developed power is converted into energy usable, i.e. being used to actuate the receiving machine (gas compressor, in our case).

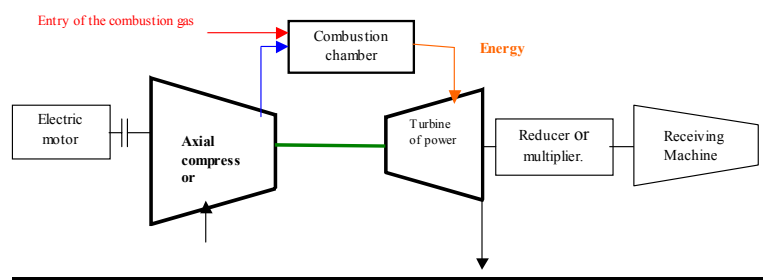

Fig. 2: Diagram of a gas turbine group

Our model of ontological representation: The role of our ontology is to describe the equipment to maintain which is a gas turbine with all its components and all its information concerning its supervision and its control. The sources of texts which are used to extract knowledge of domain, in order to build our ontology, are: experts of domain, books, scientific articles, handbooks of the manufacturers, CDs...

The model of representation of knowledge used for the representation of our ontology is based on frames.

\section{The conceptualization}

Concepts: the concepts which constitute our ontology are approximately in the number of 700 concepts of various types: classes, slots and instances.

The relations between concepts:

* Is-a: this relation allows leading to taxonomy of concepts.

* Part-of: this relation makes possible to determine subcomponents of a component.

* Produce: this relation shows that a concept (parameter) produced of other concepts (alarm, complete stop).

* Control: this relation shows the concepts controlled by another concept

* Supervise: this relation allows showing that a component is supervised by another component (instrument).

* Occur because: this relation shows that a concept « alarm/ complete stop» occurs because of other concept « causes probable».
* Have: this relation shows that a concept "causes probable" has one or more remedies.

Axioms: among the detected axioms let us quote:

* An instrument is a component.

* The gas turbine is an equipment

* If a parameter (temperature, pressure, vibration...), given by an instrument, is lower or higher than a normal value, an alarm is announced or a complete stopping of the equipment is carried out.

* A system belongs to equipment.

* An auxiliary component is a component belonging to a system.

* A basic component is a component belonging to the equipment.

* An element of component is a component belonging to a component.

* Each instrument monitors a determined component in order to give parameters of control (temperature, pressure, vibration...) on this component.

* Each Alarm is produced because of one or several probable causes.

* Each complete stop is produced because of one or several probable causes.

* Each probable cause has one or more remedies. ...

An extract of our ontology representation model:

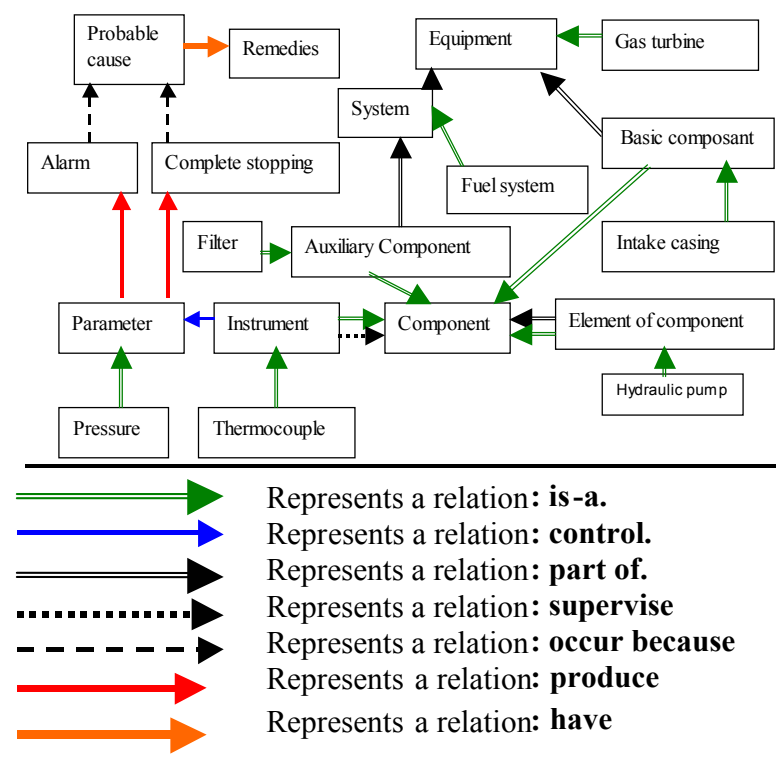

Figure 3: conceptual model of our ontology

2. The ontologization: Let us recall that an ontologization is only a conceptual representation of domain knowledge, independently of the operational uses. It is a question of transcribing knowledge in a language or paradigm of representation of ontology.

For the ontologization of our ontology, we have used the tool Protégé2000 version 3.1.1 in which knowledge is represented about frames. In the prototype which we have developed, we have represented 
approximately 700 frames of various types: classes, slots and instances. The base of knowledge is extensible, from the point of view modeling and instanciation.

Presentation of protégé-2000: Protégé is a free, open source ontology editor and knowledge-base framework. The Protégé platform supports two main ways of modeling ontologies via the Protégé-Frames and Protégé-OWL editors. Protégé ontologies can be exported into a variety of formats including $\mathrm{RDF}(\mathrm{S})$, OWL and XML Schema. Protégé is based on Java, is extensible and provides a plug-and-play environment that makes it a flexible base for rapid prototyping and application development ${ }^{[4]}$. Protégé-2000 is an integrated software tool used by system developers and domain experts to develop knowledge-based systems. Applications developed with Protégé-2000 are used in problem-solving and decisionmaking in a particular domain. Ontology in protegé2000 is structured in classes, slots, facets and axioms.

a. Classes and instances: The classes of protégé-2000 constitute a taxonomic hierarchy. The root of this hierarchy is the Thing class. Protegé-2000 supports the multiple heritages between the classes.

b. Slots: The slots in protégé-2000 are special frames attached to the classes to define their attributes.

c. Facet: The facets permit to specify the constraints on the values of slots. These constraints include the cardinalities of the slots and the restrictions on the types of values.

Protecgé-2000 allows the capture of structured data by using windows of acquisition of knowledge to acquire information of the instances. When the user defines a class, it attaches slots to him. The protégé2000 system automatically generates a window to acquire the instances of this class. This window can be personalized by the user ${ }^{[5]}$.

\section{The class hierarchy of our ontology:}

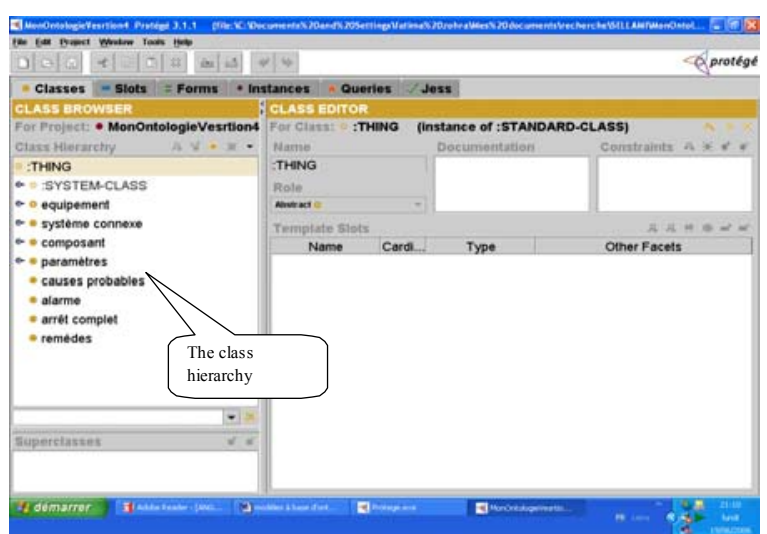

\section{Example of definition of the slots:}

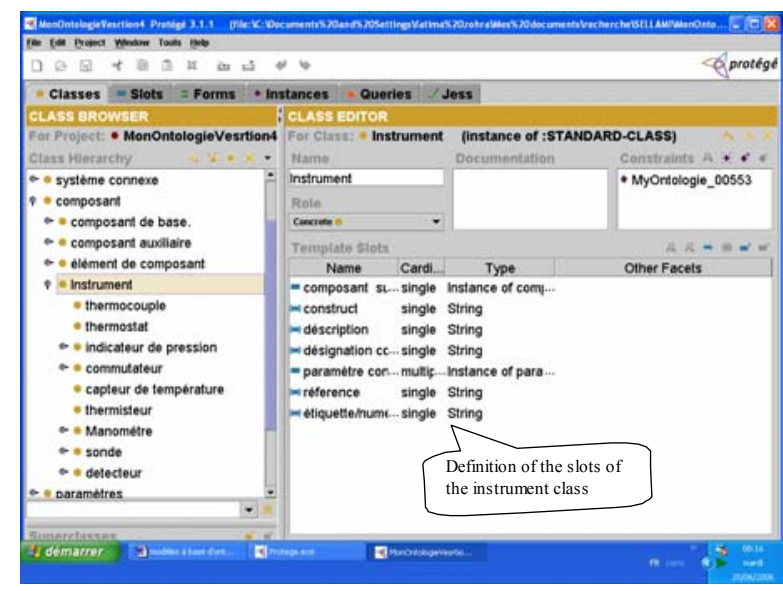

\section{Example of instances of the thermocouple class:}

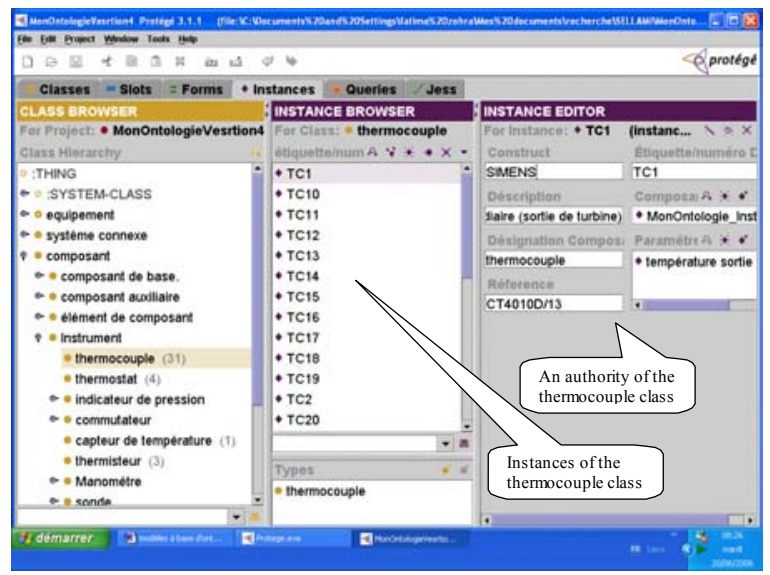

Example of definition of the slot « composant du system »:

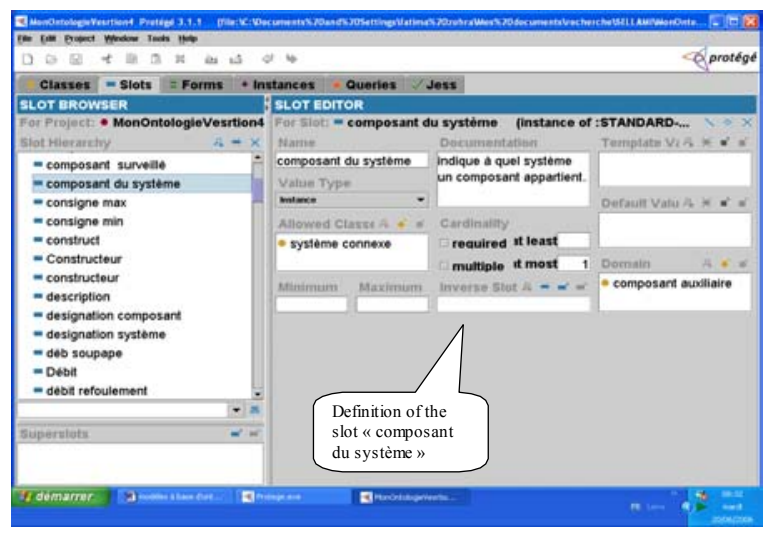

Evaluation

Qualitative evaluation: Two types of evaluation are considered:

* Evaluation by the users (with criteria based on the satisfaction of the users) 
* Strategic evaluation by the manager (with criteria based on the income on the investment)

Among the criteria of such an evaluation: facility of recovery of information, adequacy of recovered information, possible confidentiality of information...

From a technical point of view, the transfer of know-how inside the company seems to have an obvious benefit, but the real transfer depends on a real use of the knowledge capitalized on the level of the company (changes of the practices in individual and collective work).

Quantitative evaluation: Evaluation by the experts of domain in term of cover of the domain considered.

* The validation of our ontology by the experts showed that our ontology covers the entire domain considered. Our ontology is extensible from the point of view modeling and instanciation.

* The steps of validation and evaluation are iterative and must be continued at the time of the use in concrete situation.

\section{CONCLUSION}

We tried in this work to present an ontology in the domain of industrial maintenance. After the stage of conceptualization, we used the tool protégé2000 to represent our ontology in a form easy to handle by the machine. The goal of our work was thus the conceptualization and the ontologization of ontology in the industrial domain. This ontology can be used in several applications. It is intended mainly at a community of experts in the domain of the maintenance of the industrial process using the gas turbines.

\section{REFERENCES}

1. Solari, J.J., 2004. OWL Web Ontology Langage Use Cases and Requirements. http://www.yoyodesign.org/ doc/ w3c/ web ontreq- 20040210/

2. Napoli, A., D. Léonard, D. Colnet, G. Masini and K. Tombre, 1991. Les langages à objets. InterEditions Mai.

3. Furs, F., 2003. L'operationalisation des ontologies. http://www. afia. polytechnique. fr/ plate forme2003/ Articles/ RJCIA/ rjcia- 03-Furst.ps>

4. http://protege.stanford.edu/

5. Sellami, M., 2002. Projet MIND : Rapport de synthèse. LRI Annaba.

6. Natalya F. Noy and al. Ontology Development 101: A Guide to Creating Your First Ontology. http://protege.stanford.edu/publications/ontology development/ontology101-noy-mcguinness.html.

7. A Glossary of Ontology Terminology http://www-ksl-svc.stanford.edu:5915/doc/frameeditor/glossary-of-terms.html.

8. Frédéric FÜRST, « Contribution à l'ingénierie des ontologies : une méthode et un outil d'opérationalisation », thèse de docteur de l'université de nantes. 25 Novembre 2004. 